\title{
Maternal, Fetal, and Placental Selectins in Women With Pre-eclampsia; Association With the Renin-Angiotensin-System
}

\author{
Hiten D. Mistry ${ }^{1 *}$, Melissa V. Hott Ogalde ${ }^{1}$, Fiona Broughton Pipkin ${ }^{1}$, Geneviève Escher ${ }^{2,3}$ \\ and Lesia O. Kurlak ${ }^{1}$ \\ ${ }^{1}$ Department of Obstetrics and Gynaecology, University of Nottingham, Nottingham, United Kingdom, ${ }^{2}$ Department of \\ Nephrology and Hypertension, University of Bern, Bern, Switzerland, ${ }^{3}$ Department of Biomedical Research, University of \\ Bern, Bern, Switzerland
}

Selectins [endothelial $(E)$, platelet $(P)$, and leucocytes $(L)$ ] are a class of cell adhesion molecules, stimulated in response to inflammation. Pre-eclampsia is characterized by inflammation, and angiotensin II is pro-inflammatory. We hypothesized that circulating maternal and fetal concentrations and placental expression of selectins would be increased in women with pre-eclampsia and would be associated with the angiotensin receptors (AT1R and AT2R). Maternal and fetal blood and placental tissue was collected at delivery from White European normotensive controls $(n=17)$ and women with pre-eclampsia $(n=17)$. Soluble $(\mathrm{s}) \mathrm{E}-, \mathrm{P}$ - and L-selectin protein concentrations were measured by ELISA and placental protein expression was examined by immunohistochemistry. Maternal sE-selectin concentrations were increased in pre-eclampsia $(P<0.001)$; conversely fetal sE- and SP-selectin levels were lower in pre-eclampsia $(P<0.05$ for both). Staining was mainly localized to the syncytiotrophoblast for all selectins. E-selectin expression was increased, while P-selectin was decreased in placental from pre-eclampsia $(P<0.05$ for both); no differences were observed for $L$-selectin expression. Both $E$ - and $L$-selectin were positively correlated $(P<0.008 ; P<0.02)$ with AT2R placental expression, whilst P-selectin was negatively associated with AT1R $(P<0.005)$, all only in the pre-eclampsia group. This novel study reports maternal, fetal and placental expression of selectins in pre-eclampsia. The increased E-selectins reflect the endothelial dysfunction, characteristic of pre-eclampsia. In contrast, the reduced P-selectins and the positive association of placental AT2Rs with both E-and L-selectin in pre-eclampsia could be a protective mechanism to limit the endothelial dysfunction.

Keywords: selectins, angiotensin receptors, endothelial dysfunction, pre-eclampsia, inflammation

\section{INTRODUCTION}

Pre-eclampsia is a pregnancy-specific syndrome characterized by de novo hypertension and proteinuria after 20 weeks gestation (1). It affects between 2 and $8 \%$ of all pregnancies and is a major cause of both maternal and perinatal morbidity and mortality worldwide $(2,3)$. The consequences of pre-eclampsia are not restricted to pregnancy, with both mothers and their 
offspring being at increased in risk of cardiovascular, metabolic and renal disease in later life $(4,5)$. The etiology of pre-eclampsia remains unknown, but endothelial dysfunction due to abnormal placentation, oxidative stress, extensive leucocyte activation and inappropriate activation of the renin-angiotensin system (RAS) appear to be key contributors (6-10). The endothelium is the main regulator of vascular homeostasis. In addition to contributing to vasodilation, the intact endothelium also has antiaggregatory, anti-inflammatory and anti-proliferative properties (11). Hypertension is associated with endothelial dysfunction, which in turn results from a dysregulation of locally-acting mediators, such as the vasodilators nitric oxide, and prostacyclin and the vasoconstrictor endothelin, and angiotensin II (Ang II)

The RAS is an important regulator of blood pressure and is one of the first hormonal systems to recognize pregnancy (10). Moreover, the RAS also plays a role in various pathological situations involving regulation of cell proliferation, inflammation, oxidative stress, and angiogenesis $(12,13)$. We have previously demonstrated a disrupted placental RAS in pre-eclampsia with the angiotensin II type 1 receptor (AT1R), activation of which results in vasoconstriction, being more highly expressed in placenta from women with pre-eclampsia (14).

Selectins are type 1 transmembrane proteins and are involved in leucocyte trafficking, mediating the initial tethering and rolling interactions between blood and lymphatic organs via the endothelium (15). Selectins were so named because of their ability to selectively bind carbohydrate moieties via their ligand P-selectin glycoprotein ligand-1 (PSGL-1) (15). There are three types of selectins, endothelial E-, platelets P- and leucocyte L-selectins; CD62E, CD62P and CD62L, respectively. All 3 selectins are composed of common extracellular domains and are distinguished from one another by their variable number of consensus repeats and very different cytoplasmic tails (16). E-selectin is normally absent from resting endothelium, but is transcriptionally induced in venules of multiple organs by inflammatory mediators, such as tumor necrosis factor (TNF) and IL-1 (17). P-selectin is constitutively synthesized in both platelets and endothelial cells and stored in Weibel-Palade bodies. They are rapidly translocated to the surface upon stimulation with inflammatory mediators, such as thrombin, histamine, and platelet-activating factor (17).

The shortest selectin molecule, present in leukocytes, is Lselectin, which, in the microvasculature, facilitates neutrophil rolling and migration during inflammatory responses (18). Elevated sE-selectin levels may be indicative of endothelial cell activation and damage. In addition to the conventional roles described above, the selectins play an essential role in human implantation; L-selectin and its ligands participate in the adhesion of the blastocyst to the endometrium at the maternal-fetal interface (19). Moreover, P- and E-selectins are also involved in immune recognition between maternal decidua and the embedded embryo, as well as trophoblast migration within decidual spiral arterioles (19). Reduced expression of all selectins has been reported in both decidual cells in uterine decidual stroma and in the placenta (cytotrophoblasts and syncytiotrophoblasts) of early miscarriages (6-11 weeks) compared to controls (terminations for social reasons) (20).
Concentrations of both soluble (s) E- and P-selectins have been reported to be increased in patients with chronic hypertension with the most severe endothelial dysfunction (21). Previous studies have reported elevated maternal plasma sEselectin concentrations in women with pre-eclampsia, both at the time of delivery (22-26) and also prior to clinical diagnosis (22). However, the available data concerning both sP- and sL-selectins $(22,23,26,27)$ are conflicting.

Associations between the RAS and the selectins have previously been reported: Ang II is a potent stimulus to angiogenesis, both directly and by supplying angiogenic factors by platelet aggregation (28) and inducing the expression of Pselectin on platelets, while lessening its expression in endothelial cells (29). In addition, platelets express AT1R on their surface, and AT1R antagonists reduce P-selectin expression in platelets (30) and inhibit P-selectin-mediated platelet adhesion to the microvessels (31). Ang II also acts directly on naive $\mathrm{T}$ cells inducing upregulation of L-selectin via the AT1R (32). Finally, Ang II treatment significantly increased E-selectin mRNA expression in vascular endothelial cells collected from lung tissues in a mouse model of pulmonary metastasis (33).

We hypothesized that both soluble and placental expression of selectins would be increased in women with pre-eclampsia reflecting the endothelial dysfunction and inflammation characteristic of the syndrome. These selectins would also be associated with RAS receptors, contributing to the mechanistic changes associated with pre-eclampsia. This study therefore aimed to measure both soluble and placental expression of all selectins in matched maternal, fetal, and placental samples from normotensive control women and women with pre-eclampsia.

\section{MATERIALS AND METHODS}

\section{Subjects and Sample Collection}

The study population consisted of 17 normotensive control women and 17 women with pre-eclampsia, collected over a period of 18 months (Table 1). Nottingham University Hospital Ethics Committee approved the investigations and written informed consent to participation was obtained from each woman. Cases were defined on admission with a clinical diagnosis of pre-eclampsia, defined as a systolic blood pressure of $\geq 140 \mathrm{~mm} \mathrm{Hg}$ and diastolic pressure (Korotkoff $\mathrm{V}$ ) of $\geq$ $90 \mathrm{~mm} \mathrm{Hg}$ on 2 occasions after 20 weeks' gestation in a previously normotensive women and proteinuria $>300 \mathrm{mg} / \mathrm{L}, 500 \mathrm{mg} /$ day or $2+$ on dipstick analysis of midstream urine (MSU) if $24-\mathrm{h}$ collection result was not available (1). Although samples were collected under the criteria defined originally (1), they still fit the recently updated definition (34). For subgroup analysis, the pre-eclampsia group was further split by early- (diagnosis $\leq$ 34 weeks) and late- (diagnosis $>34$ weeks) onset pre-eclampsia (35). Medical and obstetric histories, including delivery data, were obtained for each woman. The birthweight centile for each baby was computed, correcting for gestation age, sex, maternal parity, and body mass index (BMI) (36). Venous blood samples were collected before delivery and umbilical venous (fetal) blood were collected immediately after and processed as previously described (6); samples were stored in aliquots at $-80^{\circ} \mathrm{C}$ until 
TABLE 1 | Clinical and obstetric data of subject groups ${ }^{*}$

\begin{tabular}{lcc}
\hline Parameter & NC $(\boldsymbol{n}=\mathbf{1 7})$ & PE $(\boldsymbol{n}=\mathbf{1 7})$ \\
\hline Age (yrs) & $28.2 \pm 7.2$ & $31 \pm 6.5$ \\
Booking body mass index $\left(\mathrm{Kg} / \mathrm{m}^{2}\right)$ & $26.4 \pm 5.5$ & $24.8 \pm 3.5$ \\
Smoking status & & \\
$\quad$ Non-smoker & $9(53)$ & $11(65)$ \\
$\quad$ Smoker & $8(47)$ & $6(35)$ \\
Parity & & \\
$\quad$ Nulliparous & $11(65)$ & $10(59)$ \\
$\quad$ Multiparous & $6(35)$ & $7(41)$ \\
Max. systolic blood pressure & $114 \pm 3.8$ & $155 \pm 3.8^{\dagger}$ \\
(mmHg) & $75 \pm 2.1$ & $97 \pm 5.0^{\dagger}$ \\
Max. diastolic blood pressure & & $1.0[0.3,9.4]$ \\
(mmHg) & - & $37.7 \pm 1.8$ \\
Proteinuria (g/L) Median [min, max] & $40.0 \pm 1.0$ & $2993 \pm 735^{\dagger}$ \\
Gestational age at delivery (Wks) & $3439 \pm 498$ & $13(76)$ \\
Mean birthweight (g) & $27.9[17.5,66.8]$ & $35.1[8.6,76.3]$ \\
Corrected birthweight centile & $4(24)$ & $6(35)$ \\
Caesarean section & - & \\
Early-onset PE & &
\end{tabular}

*Data represented as means $\pm S D$ or median [IQR] as appropriate, except for smoking status, parity and Caesarean sections and early-onset $P E$, which are shown as number (percentage). NC, normotensive control; $P E$, pre-eclampsia; $B M I$, body mass index. ${ }^{\dagger} P$ $<0.05$ between normotensive controls and women with pre-eclampsia.

analysis. In addition, placental tissue samples were collected from a standardized location midway between cord insertion and placental border and processed for immunohistochemistry as previously described (6).

\section{Soluble Selectin Assays}

Soluble (s) E-, P-, and L-selectin were measured in maternal and fetal EDTA plasma using ELISAs (KA0116, KA0548, and KA0117, respectively; Abnova, Taiwan) following the manufacturers' instructions. Plasma samples were diluted 1:100, run in duplicate and blinded to outcome group. Intra- and interassay variations were: sE-selectin: 5.4 and 6\%; sP-selectin: 5.5 and 6.1\%; sL-selectin: 3.7 and $4.2 \%$.

\section{Placental Selectin Protein Expression/Localization}

Immunohistochemical analysis was performed as previously described (37), using antibodies to E-selectin (mouse monoclonal, BBA16; $5 \mu \mathrm{g} / \mathrm{mL} ; \quad$ R\&D Systems), P-selectin (mouse monoclonal, BBA30; $10 \mu \mathrm{g} / \mathrm{mL} ; \mathrm{R} \& \mathrm{D}$ Systems) and L-selectin (rabbit polyclonal, GTX59778; $0.1 \mu \mathrm{g} / \mathrm{mL}$; GeneTex Inc.). All slides were assessed by the same observer, blinded to pregnancy outcome. Quantification was performed as described previously (12), using the Positive Pixel Algorithm of Aperio ImageScope software; a visual check was also performed to establish localization of staining. The AT1R and AT2R protein expression analysis was performed as previously reported (14).

\section{Statistical Analysis}

All tests were performed using SPSS version 24. Summary data are presented as means \pm standard deviation (SD) or median and interquartile range (IQR) as appropriate. The KolmogorovSmirnov test indicated the experimental data were not normallydistributed. The Kruskal-Wallis test followed by Mann-Whitney $U$-test was used for multiple group analysis; the Wilcoxon paired tests were used for maternal and fetal selectin concentrations. Potential associations between continuous data were tested using Spearman's Rho correlation tests. The null hypothesis was rejected where $P<0.05$.

\section{RESULTS}

\section{Subjects}

Demographics and clinical characteristics of the participants are summarized in Table 1. By definition, blood pressures were significantly increased $(P<0.05)$ and significant proteinuria was present in the pre-eclampsia group. Birthweights were also lower in the women who suffered from pre-eclampsia. The groups were matched for maternal age, BMI, smoking status, parity, and gestational age at delivery.

\section{Soluble Selectin Concentrations in Maternal and Fetal Plasma}

Concentrations of all soluble selectins are summarised in Figure 1. Maternal sE-selectin was increased in pre-eclampsia compared to controls $(P<0.05$; Figure 1A). No significant differences were observed for sP- or sL-selectins $(P>0.05$ for both; Figures 1B,C). In contrast, in the fetus, both sE- and sPselectin concentrations were lower in the pre-eclampsia group $(P$ $<0.05$ for both; Figures 1A,B), but did not differ for sL-selectin $(P>0.05$; Figure 1C).

When comparing paired maternal-fetal samples, both sE- and sP-selectin concentrations were increased in fetal compared to maternal plasma only in the normotensive group $(P<0.05$ for both; Figures 1A,B); fetal plasma from women who had preeclampsia remaining similar to their paired maternal plasma $(P>$ 0.05). Conversely, fetal sL-selectin concentrations were reduced compared to their paired maternal samples in both groups $(P<$ 0.05; Figure 1C).

When sub-grouped by early-/late-onset pre-eclampsia, only maternal sE-selectin concentrations were raised in plasma from early-onset $(n=6)$ pre-eclampsia $(P=0.003)$, compared to both late-onset $(n=11)$ pre-eclampsia $(P=0.01)$ and normotensive controls $(P=0.002$; Figure 1D). No differences were seen for either sP- or sL-selectin concentrations $(P>0.05$ for both; data not shown).

Ratio between maternal and fetal sE-, sP- and sL-selectin was calculated (Figure 2). Significantly increased ratios were observed for both sE- $(P=0.001)$ and $\mathrm{sP}^{-}(P=0.02)$ selectin in pre-eclampsia, compared to normotensive controls. No differences were observed for sL-selectin $(P>0.05)$.

\section{Placental Selectin Protein Expression}

Placental expression of all 3 selectins was determined and localization was mainly in the syncytiotrophoblast layer, 


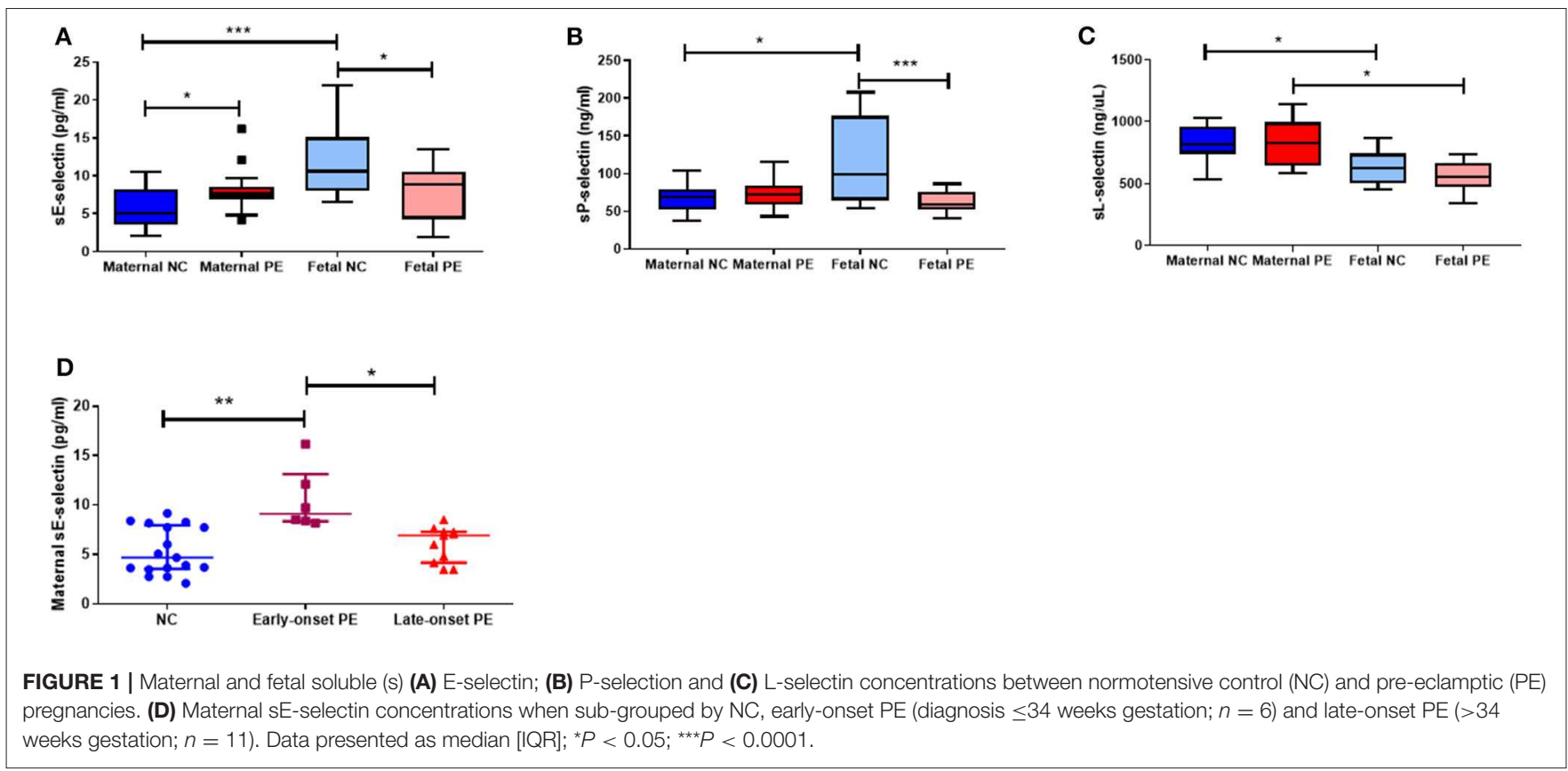

stromal areas and some fetal vessel staining (Figure 3). Placental E-selectin expression was increased in pre-eclampsia (median [IQR], positivity; normotensive controls: 0.56 [0.51, 0.65]; pre-eclampsia: $0.71[0.60,0.80] ; \quad P=0.008$; Figure 3A), whereas P-selectin was reduced in pre-eclampsia (normotensive controls 0.47 [0.34, 0.59]; pre-eclampsia 0.35 $[0.22,0.41] ; \quad P=0.008$; Figure 3B). E-selectin expression levels were raised $(P=0.002)$, while P-selectin expression was reduced in both the early- and late-onset pre-eclampsia groups, compared to normotensive control samples $(P<0.05$; Figures 3D,E).

Although no differences were observed between controls and pre-eclamptic women for L-selectin $(P>$ 0.05 ; Figure 3C), when sub-grouped by onset of preeclampsia, a significant difference again became apparent the expression being increased in early-onset pre-eclampsia group expression compared to both normotensive controls $(P=0.008)$ and late-onset pre-eclampsia $(P=0.002$; Figure 3F).

\section{Association of Placental Expression of Selectins With AT1R and AT2R}

We compared placental expression of selectins with previously measured AT1R and AT2R placental protein expression. In the pre-eclampsia samples, but not those from normotensive women, placental expression of both E-selectin $(r=0.55 ; P=0.008$; Figure 4A) and L-selectin $(r=0.61 ; P=0.02 ;$ Figure 4B) positively correlated with AT2R expression. In contrast, again only in the pre-eclampsia group, P-selectin displayed a negative association with AT1R expression $(r=0.68 ; P=0.005$; Figure 4C).

\section{DISCUSSION}

This is the first study to present data on all selectins in paired maternal/fetal plasma together with their placental expression in women with and without pre-eclampsia. It helps clarify the conflicting data found previously in relation to P- and L-selectin in pregnancy and pre-eclampsia.

The increased maternal sE-selectin in pre-eclampsia is in accordance with previous reports $(22,23,25,26)$. We have now shown that there is also increased placental E-selectin, reflecting the overall endothelial dysfunction associated with preeclampsia. Furthermore, the markedly increased concentrations of maternal sE-selectin, found only in the early-onset preeclampsia group (Figure 1D), presumably reflect the severity of the syndrome, as has been suggested by others (24). This is further supported by the finding that sE-selectin is increased prior to clinical diagnosis of pre-eclampsia $(22,38)$. Moreover, Chen et al., have shown that maternal sE-selectin was not associated with all cases of preterm birth, but specifically only when complicated by pre-eclampsia (39). The increased placental E-selectin expression we have shown corresponds with increased maternal sE-selectin and suggests that the placental dysfunction contributes to the endothelial damage in pre-eclampsia. Shaw et al., reported increased E-selectin mRNA expression in endothelial cell HUVECs and HEECs after incubation with maternal perfusates obtained from placenta of women with preeclampsia (40), which might amplify the underlying difference.

The interesting finding of reduced fetal sE-selectin in preeclampsia could either be a protective adaptation to limit the endothelial damage to the fetus, or reflects an underlying inability to synthesize E-selectin. Since E-selectin can be induced on fetal endothelial cells to the same extent as adult vessels by 32 weeks' gestation $(17,41)$ and the gestational age of the early-onset PE 

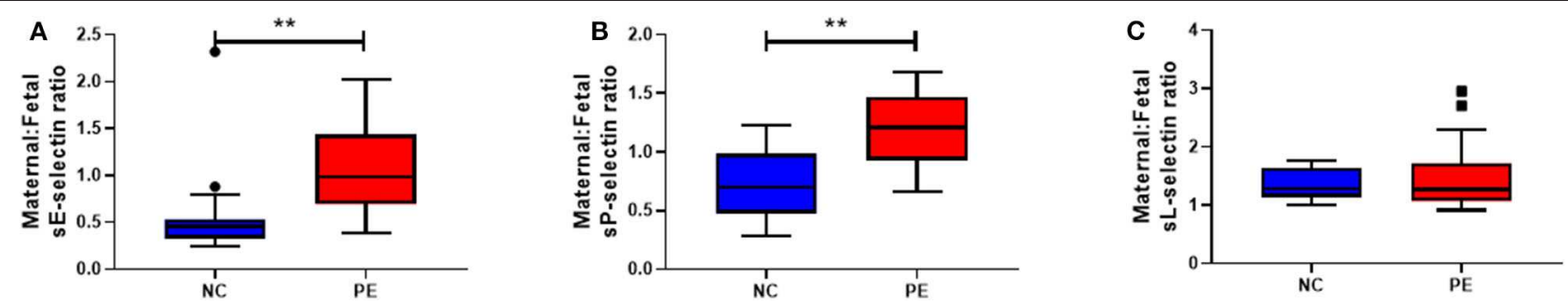

FIGURE 2 | Maternal:fetal ratios of soluble (s) (A) E-selectin; (B) P-selection and (C) L-selectin concentrations between normotensive control (NC) and pre-eclamptic (PE) pregnancies. Data in all box plots are presented as median [interquartile ranges]; ${ }^{\star \star} P<0.001$.
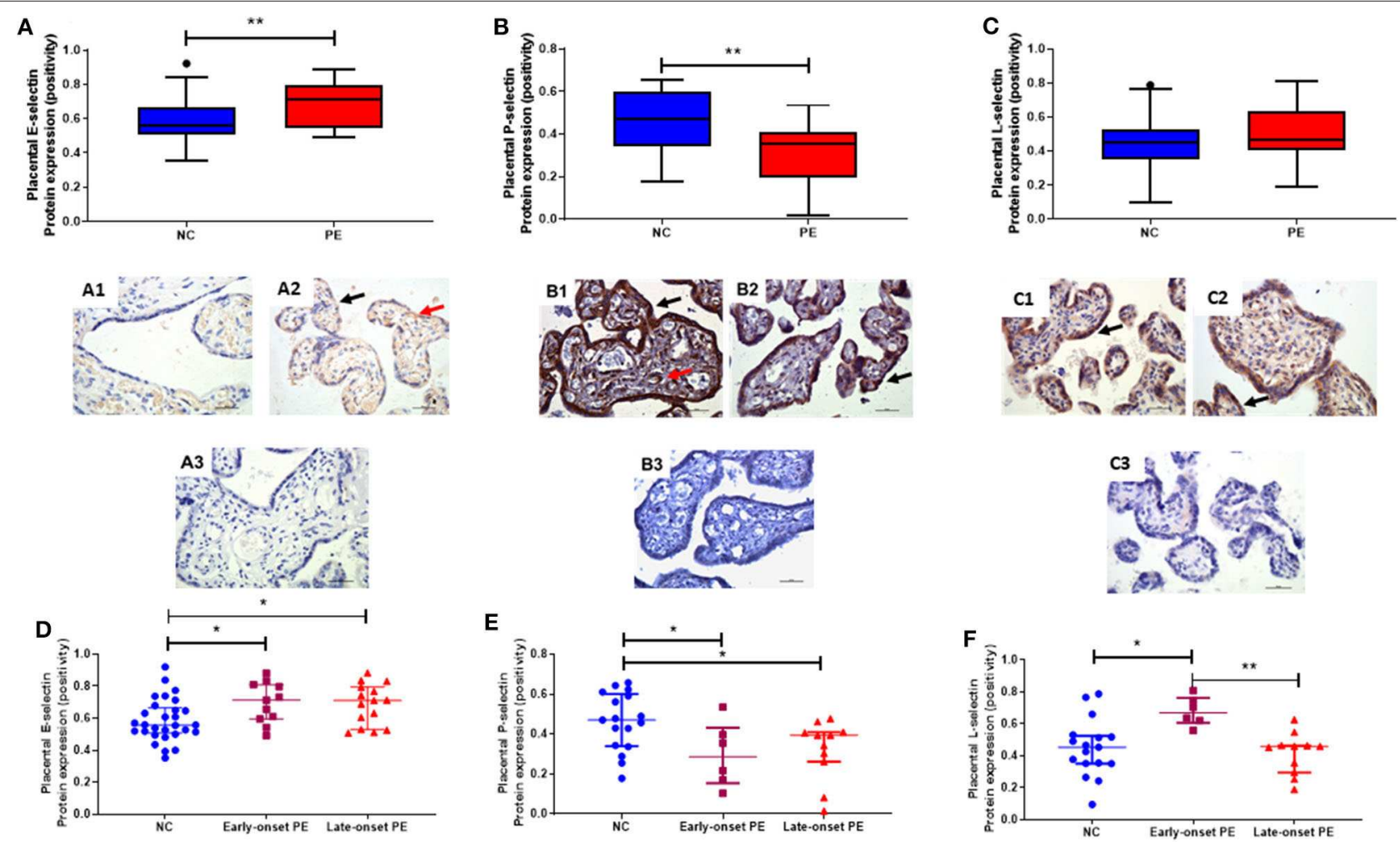

FIGURE 3 | Localisation and quantification of placental (A) E-selectin; (B) P-selectin and (C) L-selectin in placenta from normotensive controls (NC (A1, B1, \& C1); $n$ $=17$ ) and pre-eclampsia (PE (A2, B2, \& C2); $n=17)$. Negative controls for each selectins are shown in A3, B3, \& C3 respectively. In photomicrographs, positive cells appear in brown; magnification $\times 400$. Scale bars represent $100 \mu \mathrm{m}$. Protein expression was localized to the syncytiotrophoblast layer (black arrows) and some fetal vessels (red arrows). (D) E-selectin; (E) P-selectin and (F) L-selectin expression in placenta, when sub-grouped by NC, early-onset PE (diagnosis $\leq 34$ weeks gestation; $n=6$ ) and late-onset PE (>34 weeks gestation; $n=11)$. Data in all box plots are presented as median [interquartile ranges]; ${ }^{*}<0.05$; ${ }^{\star \star} P<0.001$.

group was nearer 34 weeks, this supports the view that the difference is attributable to the pre-eclampsia pathology and not an immaturity of development. sE-selectin also contributes to homing in the endothelial progenitor cells and promotes tube formation in microvasculature as a repair mechanism (42). Given that there is reduced tubule formation in HUVECS isolated from pre-eclamptic pregnancies (43), this further suggests that the functional capacity of fetal endothelial cells is impaired in pre-eclampsia.

Nevertheless, the positive correlation with placental AT2R expression only in the pre-eclamptic samples supports the hypothesis of a protective mechanism; AT2R forms part of the "protective" arm of the RAS and is strongly upregulated following tissue damage such a vascular and neuronal injury (44). AT2R confers this role through its anti-inflammatory, anti-fibrotic and anti-apoptotic properties. Moreover, in lung tissues, which have many parallels with the placenta, Ang II treatment significantly increased E-selectin mRNA expression in vascular endothelial cells, further supporting the association (33).

We did not identify any differences in maternal sP-selectin between groups, which is in contrast to a previous study, which reported increased levels in pre-eclampsia $(23,26)$. A potential explanation for this difference lies in the later gestation age at sampling in our study when compared to the previous study. The 

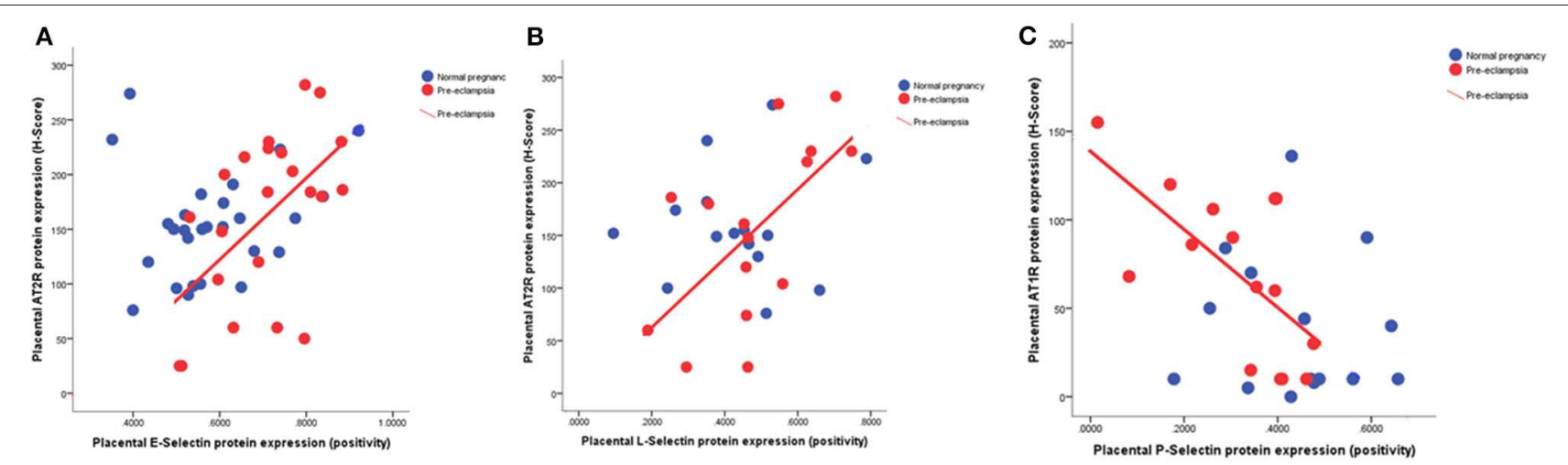

FIGURE 4 | Scatter plots illustrating the positive relationship between (A) placental E-selectin $(r=0.55 ; P=0.008)$ and $(\mathbf{B}) \mathrm{L}$-selectin $(r=0.61 ; P=0.02)$ with angiotensin type 2 receptor (AT2R) and (C) the negative association between placental P-selectin $(r=0.68 ; P=0.005)$ with angiotensin type 1 receptor (AT1R).

lack of differences in sP-selectins between groups could have been due to our not measuring platelet sP-selectin levels. Nevertheless, by using whole plasma, we were able to measure all 3 selectins.

As with sE-selectins, the lower fetal P-selectin concentrations in the pre-eclamptic group could be a protective mechanism to limited endothelial damage to the fetus. The reduced placental P-selectin expression in pre-eclampsia could also contribute to lower fetal sP-selectin. In addition, the decreased P-selectin could also be explained by the known reduction in platelet numbers in pre-eclampsia (34). Furthermore, the inverse relationship with AT1R, suggests this is independent of AT1R expressed on platelets. Ang II can increase P-selectin expression within the vascular wall (45) and thus increase atherosclerotic plaque progression. Therefore, the reduced placental P-selectin expression in pre-eclampsia could be a feedback mechanism to limit this damage and is supported by this negative correlation with AT1R.

Inspection of Figures 2A,B led us to suggest that the ratio between maternal and fetal sE- or sP-selectin might differ between normotensive and pre-eclamptic women, and in early and late-onset pre-eclampsia. As Figure 3 shows, this is indeed the case, again emphasizing a possible protective mechanism.

The lack of differences observed between groups in sLselectin in plasma may be because differences are only seen in specific cell types, for example the decreased levels in preeclampsia reported in neutrophils, T-cells, and monocytes (46). However, although not significant, there did appear to be a trend toward reduced maternal sL-selectin concentrations in the earlyonset pre-eclamptic group. This is in line with previous studies where the majority of the pre-eclamptic samples measured were from early-onset pre-eclampsia $(23,47)$. To investigate this fully, future studies will examine whether any differences are observed when leukocytes and platelets are isolated and analyzed to confirm if the differences in this study are confirmed, or were masked by measuring in whole plasma. In contrast, there is increased placental expression in placenta from the earlyonset pre-eclampsia group, which is not reflected in parallel increases in the maternal or fetal circulation. This again suggests a possible protective mechanism, further supported by the positive association seen with placental AT2R expression in the preeclamptic group. It has been suggested that these lower levels result in increased leukocyte activation as seen in some chronic inflammatory states (e.g., chronic artery disease (48)). We can thus speculate that the increased placental L-selectin in earlyonset pre-eclampsia could lead to increased leucocyte activation and therefore atherosis in these placenta.

To conclude, this study is the first to report matched maternal, fetal and placental expression of all 3 selectins in pre-eclampsia. The increased E-selectins reflect the endothelial dysfunction, characteristic of pre-eclampsia. In contrast, the reduced $\mathrm{P}$ selectins and the positive association with the placental AT2R with both E-and L-selectin in pre-eclampsia could be a protective mechanism to limit the endothelial dysfunction. Due to the roles of the selectins, this observational study further supports the importance of endothelial cells, platelets, and leucocytes in the pathogenesis of pre-eclampsia.

\section{DATA AVAILABILITY STATEMENT}

The datasets generated for this study are available on request to the corresponding author.

\section{ETHICS STATEMENT}

The studies involving human participants were reviewed and approved by Nottingham University Hospital Ethics Committee. The patients/participants provided their written informed consent to participate in this study.

\section{AUTHOR CONTRIBUTIONS}

HM, LK, MO, and GE developed and performed measurement techniques, analyzed data, and wrote the manuscript. LK and HM collected samples and clinical information. FB directed the project, analyzed data, and assisted with writing the manuscript. All authors provided critical reviews of the manuscript. 


\section{ACKNOWLEDGMENTS}

We thanks Heidi Jamin and Beatrice Rohrbach for running the ELISA assays and all the women who participated in the study.
This work was supported by the British Heart Foundation Basic Science Intermediate Basic Science Fellowship [FS/15/32/31604 to HM]. Some parts of the data, in an adapted form from this manuscript has been published as a conference paper (49).

\section{REFERENCES}

1. Brown MA, Lindheimer MD, de Swiet M, Van Assche A, Moutquin JM. The classification and diagnosis of the hypertensive disorders of pregnancy: statement from the International Society for the Study of Hypertension in Pregnancy (ISSHP). Hypertens Pregnancy. (2001) 20:IXXIV. doi: 10.3109/10641950109152635

2. Steegers EA, von Dadelszen P, Duvekot JJ, Pijnenborg R. Pre-eclampsia. Lancet. (2010) 376:631-44. doi: 10.1016/S0140-6736(10)60279-6

3. Duley L. The global impact of pre-eclampsia and eclampsia. Semin Perinatol. (2009) 33:130-7. doi: 10.1053/j.semperi.2009.02.010

4. Bellamy L, Casas JP, Hingorani AD, Williams DJ. Pre-eclampsia and risk of cardiovascular disease and cancer in later life: systematic review and meta-analysis. BMJ. (2007) 335:974. doi: 10.1136/bmj.39335.385301.BE

5. Mistry HD, Kurlak LO, Mansour YT, Zurkinden L, Mohaupt MG, Escher G. Increased maternal and fetal cholesterol efflux capacity and placental CYP27A1 expression in preeclampsia. J Lipid Res. (2017) 58:118695. doi: 10.1194/jlr.M071985

6. Mistry HD, Wilson V, Ramsay MM, Symonds ME, Broughton Pipkin F. Reduced selenium concentrations and glutathione peroxidase activity in pre-eclamptic pregnancies. Hypertension. (2008) 52:881-8. doi: 10.1161/HYPERTENSIONAHA.108.116103

7. Poston L. Endothelial dysfunction in pre-eclampsia. Pharmacol Rep. (2006) 58 Suppl:69-74.

8. Poston L, Igosheva N, Mistry HD, Seed PT, Shennan AH, Rana S, et al. Role of oxidative stress and antioxidant supplementation in pregnancy disorders. Am J Clin Nutr. (2011) 94(6 Suppl):1980S-5S. doi: 10.3945/ajcn.110.001156

9. Campello E, Spiezia L, Radu CM, Dhima S, Visentin S, Valle FD, et al. Circulating microparticles in umbilical cord blood in normal pregnancy and pregnancy with preeclampsia. Thromb Res. (2015) 136:42731. doi: 10.1016/j.thromres.2015.05.029

10. Broughton Pipkin F. The renin-angiotensin system in pre-eclampsia. In: Lyall F, Belfort M, editors. Pre-Eclampsia. Etiology Clinical Practice. Cambridge: Cambridge University Press (2007) 78-91.

11. Ross R. Atherosclerosis-an inflammatory disease. N Engl J Med. (1999) 340:115-26. doi: 10.1056/NEJM199901143400207

12. Williams PJ, Mistry HD, Innes BA, Bulmer JN, Broughton Pipkin F. Expression of AT1R, AT2R and AT4R and their roles in extravillous trophoblast invasion in the human. Placenta. (2010) 31:448-55. doi: 10.1016/j.placenta.2010.02.014

13. Farag E, Maheshwari K, Morgan J, Sakr Esa WA, Doyle DJ. An update of the role of renin angiotensin in cardiovascular homeostasis. Anesth Analg. (2015) 120:275-92. doi: 10.1213/ANE.0000000000000528

14. Mistry HD, Kurlak LO, Broughton Pipkin F. The placental renin-angiotensin system and oxidative stress in pre-eclampsia. Placenta. (2013) 34:1826. doi: 10.1016/j.placenta.2012.11.027

15. Zarbock A, Ley K, McEver RP, Hidalgo A. Leukocyte ligands for endothelial selectins: specialized glycoconjugates that mediate rolling and signaling under flow. Blood. (2011) 118:6743-51. doi: 10.1182/blood-2011-07-343566

16. Kappelmayer J, Nagy B Jr. The interaction of selectins and PSGL-1 as a key component in thrombus formation and cancer progression. Biomed Res Int. (2017) 2017:6138145. doi: 10.1155/2017/6138145

17. Auvinen K, Jalkanen S, Salmi M. Expression and function of endothelial selectins during human development. Immunology. (2014) 143:406-15. doi: 10.1111/imm. 12318

18. Walcheck B, Moore KL, McEver RP, Kishimoto TK. Neutrophil-neutrophil interactions under hydrodynamic shear stress involve L-selectin and PSGL-1. A mechanism that amplifies initial leukocyte accumulation of P-selectin in vitro. J Clin Invest. (1996) 98:1081-7. doi: 10.1172/JCI1 18888

19. Feng Y, Ma X, Deng L, Yao B, Xiong Y, Wu Y, et al. Role of selectins and their ligands in human implantation stage. Glycobiology. (2017) 27:38591. doi: 10.1093/glycob/cwx009

20. Eskicioglu F, Lacin S, Ozbilgin K, Kose C. The role of selectins in the first trimester pregnancy loss. Ginekol Pol. (2014) 85:287-93. doi: $10.17772 / g p / 1725$

21. de la Sierra A, Larrousse M. Endothelial dysfunction is associated with increased levels of biomarkers in essential hypertension. J Hum Hypertens. (2010) 24:373-9. doi: 10.1038/jhh.2009.91

22. Carty DM, Anderson LA, Freeman DJ, Welsh PI, Brennand JE, Dominiczak AF, et al. Early pregnancy soluble E-selectin concentrations and risk of preeclampsia. J Hypertens. (2012) 30:954-9. doi: 10.1097/HJH.0b013e328352573b

23. Chaiworapongsa T, Romero R, Yoshimatsu J, Espinoza J, Kim YM, Park K, et al. Soluble adhesion molecule profile in normal pregnancy and pre-eclampsia. J Matern Fetal Neonatal Med. (2002) 12:19-27. doi: 10.1080/jmf.12.1.19.27

24. Veas CJ, Aguilera VC, Munoz IJ, Gallardo VI, Miguel PL, Gonzalez MA, et al. Fetal endothelium dysfunction is associated with circulating maternal levels of sE-selectin, sVCAM1, and sFlt-1 during pre-eclampsia. J Mat-Fetal Neon Med. (2011) 24:1371-7. doi: 10.3109/14767058.2011.556204

25. Krauss T, Azab H, Dietrich M, Augustin HG. Fetal plasma levels of circulating endothelial cell adhesion molecules in normal and preeclamptic pregnancies. Eur J Obstet Gynecol Reprod Biol. (1998) 78:41-5. doi: 10.1016/S0301-2115(98)00010-4

26. Docheva N, Romero R, Chaemsaithong P, Tarca AL, Bhatti G, Pacora $\mathrm{P}$, et al. The profiles of soluble adhesion molecules in the "great obstetrical syndromes"(). J Matern Fetal Neonatal Med. (2019) 32:211336. doi: 10.1080/14767058.2018.1427058

27. Nasrollahi S, Hoseini Panah SM, Tavilani H, Tavasoli S, Naderan M, Shoar S. Antioxidant status and serum levels of selectins in pre-eclampsia. J Obstet Gynaecol. (2015) 35:16-8. doi: 10.3109/01443615.2014.935710

28. Senchenkova EY, Russell J, Almeida-Paula LD, Harding JW, Granger DN. Angiotensin II-mediated microvascular thrombosis. Hypertension. (2010) 56:1089-95. doi: 10.1161/HYPERTENSIONAHA.110.158220

29. Amano H, Ito Y, Ogawa F, Eshima K, Suzuki T, Oba K, et al. Angiotensin II type $1 \mathrm{~A}$ receptor signaling facilitates tumor metastasis formation through P-selectin-mediated interaction of tumor cells with platelets and endothelial cells. Am J Pathol. (2013) 182:553-64. doi: 10.1016/j.ajpath.2012.10.026

30. Alexandru N, Popov D, Georgescu A. Platelet dysfunction in vascular pathologies and how can it be treated. Thromb Res. (2012) 129:11626. doi: 10.1016/j.thromres.2011.09.026

31. Ishikawa M, Sekizuka E, Yamaguchi N, Nakadate H, Terao S, Granger DN, et al. Angiotensin II type 1 receptor signaling contributes to platelet-leukocyteendothelial cell interactions in the cerebral microvasculature. Am J Physiol Heart Circ Physiol. (2007) 292:H2306-15. doi: 10.1152/ajpheart.00601.2006

32. Silva-Filho JL, Souza MC, Henriques MG, Morrot A, Savino W, Caruso-Neves $\mathrm{C}$, et al. Renin-angiotensin system contributes to naive T-cell migration in vivo. Arch Biochem Biophys. (2015) 573:1-13. doi: 10.1016/j.abb.2015.02.035

33. Ishikane S, Hosoda $\mathrm{H}$, Nojiri $\mathrm{T}$, Tokudome $\mathrm{T}$, Mizutani $\mathrm{T}$, Miura $\mathrm{K}$, et al. Angiotensin II promotes pulmonary metastasis of melanoma through the activation of adhesion molecules in vascular endothelial cells. Biochem Pharmacol. (2018) 154:136-47. doi: 10.1016/j.bcp.2018.04.012

34. Brown MA, Magee LA, Kenny LC, Karumanchi SA, McCarthy FP, Saito S, et al. The hypertensive disorders of pregnancy: ISSHP classification, diagnosis and management recommendations for international practice. Pregnancy Hypertens. (2018) 13:291-310. doi: 10.1016/j.preghy.2018.05.004

35. Tranquilli AL, Brown MA, Zeeman GG, Dekker G, Sibai BM. The definition of severe and early-onset preeclampsia. Statements from the International Society for the Study of Hypertension in Pregnancy (ISSHP). Pregnancy Hypertens. (2013) 3:44-7. doi: 10.1016/j.preghy.2012.11.001 
36. Gardosi J, Chang A, Kalyan B, Sahota D, Symonds EM. Customised antenatal growth charts. Lancet. (1992) 339:283-7. doi: 10.1016/0140-6736(92)91342-6

37. Mistry HD, McCallum LA, Kurlak LO, Greenwood IA, Broughton Pipkin F, Tribe RM. Novel expression and regulation of voltage-dependent potassium channels in placentas from women with preeclampsia. Hypertension. (2011) 58:497-504. doi: 10.1161/HYPERTENSIONAHA.111.173740

38. Chavarria ME, Lara-Gonzalez L, Garcia-Paleta Y, Vital-Reyes VS, Reyes A. Adhesion molecules changes at 20 gestation weeks in pregnancies complicated by preeclampsia. Eur J Obstet Gynecol Reprod Biol. (2008) 137:157-64. doi: 10.1016/j.ejogrb.2007.06.014

39. Chen X, Scholl TO. Maternal biomarkers of endothelial dysfunction and preterm delivery. PLOS ONE. (2014) 9:e85716. doi: 10.1371/journal.pone.0085716

40. Shaw J, Tang Z, Schneider H, Salje K, Hansson SR, Guller S. Inflammatory processes are specifically enhanced in endothelial cells by placental-derived TNF-alpha: Implications in preeclampsia (PE). Placenta. (2016) 43:18. doi: 10.1016/j.placenta.2016.04.015

41. Nussbaum C, Gloning A, Pruenster M, Frommhold D, Bierschenk S, Genzel-Boroviczeny O, et al. Neutrophil and endothelial adhesive function during human fetal ontogeny. J Leukoc Biol. (2013) 93:17584. doi: 10.1189/jlb.0912468

42. Oh IY, Yoon CH, Hur J, Kim JH, Kim TY, Lee CS, et al. Involvement of E-selectin in recruitment of endothelial progenitor cells and angiogenesis in ischemic muscle. Blood. (2007) 110:38919. doi: 10.1182/blood-2006-10-048991

43. Brodowski L, Burlakov J, Hass S, von Kaisenberg C, von Versen-Hoynck F. Impaired functional capacity of fetal endothelial cells in preeclampsia. PLoS ONE. (2017) 12:e0178340. doi: 10.1371/journal.pone.0178340

44. Namsolleck P, Recarti C, Foulquier S, Steckelings UM, Unger T. AT(2) receptor and tissue injury: therapeutic implications. Curr Hypertens Rep. (2014) 16:416. doi: 10.1007/s11906-013-0416-6
45. Tayeh MA, Scicli AG. Angiotensin II and bradykinin regulate the expression of P-selectin on the surface of endothelial cells in culture. Proc Assoc Am Physicians. (1998) 110:412-21.

46. Luppi P, Tse H, Lain KY, Markovic N, Piganelli JD, DeLoia JA. Preeclampsia activates circulating immune cells with engagement of the NF-kappaB pathway. Am J Reprod Immunol. (2006) 56:135-44. doi: 10.1111/j.1600-0897.2006.00386.x

47. Sacks GP, Studena K, Sargent K, Redman CW. Normal pregnancy and preeclampsia both produce inflammatory changes in peripheral blood leukocytes akin to those of sepsis. Am J Obstet Gynecol. (1998) 179:806. doi: 10.1016/S0002-9378(98)70254-6

48. Haught WH, Mansour M, Rothlein R, Kishimoto TK, Mainolfi EA, Hendricks $\mathrm{JB}$, et al. Alterations in circulating intercellular adhesion molecule-1 and Lselectin: further evidence for chronic inflammation in ischemic heart disease. Am Heart J. (1996) 132(1 Pt 1):1-8. doi: 10.1016/S0002-8703(96)90383-X

49. Kurlak LO, Hott-Ogalde MV, Scaife PJ, Escher G, Mistry HD. Maternal and placental selectins in women with pre-eclampsia; association with the renin-angiotensin system. Placenta. (2019) 83:e105. doi: 10.1016/j.placenta.2019.06.332

Conflict of Interest: The authors declare that the research was conducted in the absence of any commercial or financial relationships that could be construed as a potential conflict of interest.

Copyright (C) 2020 Mistry, Ogalde, Broughton Pipkin, Escher and Kurlak. This is an open-access article distributed under the terms of the Creative Commons Attribution License (CC BY). The use, distribution or reproduction in other forums is permitted, provided the original author(s) and the copyright owner(s) are credited and that the original publication in this journal is cited, in accordance with accepted academic practice. No use, distribution or reproduction is permitted which does not comply with these terms. 